\title{
Esdras MINVILLE (1896-1975)
}

Intellectuel québécois de la première moitié du XXe siècle, économiste et sociologue, lié de près au groupe catholique, nationaliste et traditionaliste

directeur de l’Écoles des Hautes Études Commerciales de Montréal

(1953)

\section{"Conditions de notre avenir"}

Un document produit en version numérique par Jean-Marie Tremblay, bénévole, professeur de sociologie au Cégep de Chicoutimi

Courriel: jean-marie tremblay@uqac.ca

Site web pédagogique : http://www.uqac.ca/jmt-sociologue/

Dans le cadre de: "Les classiques des sciences sociales" Une bibliothèque numérique fondée et dirigée par Jean-Marie Tremblay, professeur de sociologie au Cégep de Chicoutimi Site web: http://classiques.uqac.ca/

Une collection développée en collaboration avec la Bibliothèque Paul-Émile-Boulet de l'Université du Québec à Chicoutimi

Site web: http://bibliotheque.uqac.ca/ 
Cette édition électronique a été réalisée par Jean-Marie Tremblay, bénévole, professeur de sociologie au Cégep de Chicoutimi à partir de :

Esdras MINVILLE (1896-1975)

“Conditions de notre avenir".

Un article publié dans Essais sur le Québec contemporain. Essays on Contemporary Quebec. Édités par Jean-Charles Falardeau. Symposium du Centenaire de l'Université Laval, chapitre XI, pp. 231-238. Québec: Les Presses de l'Université Laval.

Monsieur Esdras Minville était un intellectuel québécois de la première moitié du XXe siècle, économiste et sociologue, lié de près au groupe catholique, nationaliste et traditionaliste. Il fut directeur de l’Écoles des Hautes Études Commerciales de Montréal

Polices de caractères utilisée :

Pour le texte: Times New Roman, 14 points.

Pour les citations : Times New Roman, 12 points.

Pour les notes de bas de page : Times New Roman, 12 points.

Édition électronique réalisée avec le traitement de textes Microsoft Word 2004 pour Macintosh.

Mise en page sur papier format

LETTRE (US letter), 8.5'’ x 11’’)

Édition numérique réalisée le 2 juin 2007 à Chicoutimi, Ville de Saguenay, province de Québec, Canada. 


\section{Esdras MINVILLE (1896-1975)}

\section{"Conditions de notre avenir"}

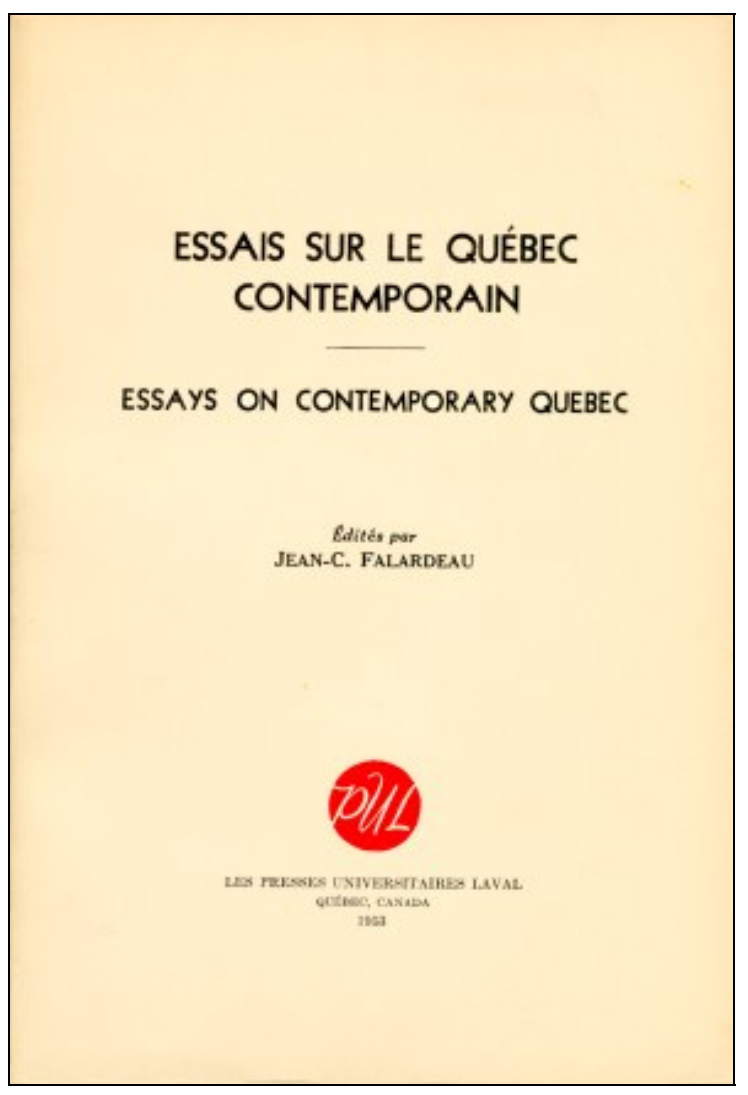

Un article publié dans Essais sur le Québec contemporain. Essays on Contemporary Quebec. Édités par Jean-Charles Falardeau. Symposium du Centenaire de l'Université Laval, chapitre XI, pp. 231-238. Québec: Les Presses de l'Université Laval. 


\section{Esdras MINVILLE (1896-1975)}

“Conditions de notre avenir".

Un article publié dans Essais sur le Québec contemporain. Essays on Contemporary Quebec. Édités par Jean-Charles Falardeau. Symposium du Centenaire de l'Université Laval, chapitre XI, pp. 231-238. Québec : Les Presses de l'Université Laval.

Afin de me mettre le mieux possible dans l'esprit de ces discussions de l'un des plus importants faits sociaux de notre milieu, j'ai cru nécessaire de retourner aux quelques ouvrages sociologiques qui ont été consacrés jusqu'ici au fait canadien-français. J'ai d'abord relu en entier le livre que le professeur Everett-C. Hughes publiait, il y a quelques années, sur une petite ville de notre province devenue, au cours des vingt-cinq ou trente dernières années, un centre industriel important. J'ai éprouvé à nouveau l'impression ressentie à la première lecture, savoir, que c'est là une sorte de photographie des reclassements qui s'effectuent dans la population et des modes nouveaux selon lesquels s'établissent les relations quand une grande industrie, appartenant à des maîtres étrangers, vient s'établir au sein d'une agglomération canadienne-française. Par son objectivité, la précision de ses méthodes, l'étendue et l'exactitude de son information, cet ouvrage demeure encore un instrument de travail nécessaire à quiconque veut comprendre le problème social comme il se pose désormais dans notre province, surtout sous l'angle des relations ethniques.

J'ai ensuite parcouru rapidement le Saint-Denis de Miner, histoire de constater encore une fois comment réagit un homme de l'extérieur au spectacle de nos manières traditionnelles de vivre, de concevoir et de pratiquer la vie sociale. Puis, je suis remonté au vieux et toujours actuel Léon Gérin : Le type économique et social des Canadiens, Aux sources de notre histoire. 
Avec Hughes, Miner et Gérin, j'ai donc relu ou feuilleté à nouveau les principales études scientifiques consacrées jusqu'ici aux problèmes sociologiques du Canada français. En tout, quatre volumes. Si l'on considère la valeur intrinsèque de chacun d'entre eux, on peut dire que c'est quelque chose, même beaucoup. Mais si l'on considère l'étendue et la complexité du phénomène à étudier, ainsi que la rapidité avec laquelle il se, modifie et se propage sous nos yeux, il faut bien admettre que c'est peu, beaucoup trop peu. Bien des enquêtes et des recherches à longue portée demeurent à entreprendre qui seraient absolument indispensables à l'élaboration d'une politique économique et d'une politique sociale adaptées aux besoins de notre population.

L'industrialisation est certainement le phénomène dominant de notre époque, la cause la plus profonde et la plus agissante de l'évolution, disons mieux, de la révolution sociale dont nous sommes à la fois les témoins et l'objet. Mais pour comprendre ce phénomène, en mesurer la portée et la gravité, il ne suffit pas de l'observer de l'extérieur et d'en décrire la marche. Il faut tâcher de le voir par le dedans, c'est-àdire ne pas se contenter de dégager les faits, de les classer, d'en deviner l'interdépendance, mais chercher à saisir la pensée qui les anime et les interpréter à partir d'une pensée plus haute, tenue elle-même comme étant la véritable loi de l'homme.

La révolution industrielle concerne tous les peuples occidentaux, c'est-à-dire tous les peuples dont le régime social est en somme issu de la même conception générale de l'homme et de ses relations avec la société. Elle les concerne tous par la diffusion même des techniques qui, en révolutionnant le travail lui-même et la production, ne peuvent pas ne pas modifier l'attitude des hommes envers l'une des données les plus impératives de leur existence. Elle les concerne surtout par l'esprit qui, jusqu'ici, a animé l'usage des techniques et qui, lui, transforme le régime de travail, celui de la famille, de la propriété, les modes de vie, tendant du même coup à modifier jusqu'aux modes de pensée. Au bout de l'évolution en cours, il peut y avoir ou bien un homme libéré, c'est-à-dire soustrait aux servitudes de la matière, ou un robot, c'est-à-dire un homme assujetti aux techniques elles-mêmes. Dans le premier cas, le progrès économique aura été une conquête ; dans le second, une immense tromperie. Tous les peuples de même filiation culturelle, humaniste et chrétienne, ont donc à fournir le même effort 
de pensée s'ils veulent profiter des avantages que les progrès scientifiques leur apportent, sans perdre le sens des valeurs de vie infiniment plus précieuses qu'ils doivent à leur civilisation traditionnelle. Humaniser les techniques, humaniser l'économie, redéfinir la société à partir de l'homme et redéfinir l'homme à partir de sa loi : tel est le devoir de l'heure.

Ce devoir s'impose aux nations occidentales, ai-je dit, pour deux raisons capitales : parce que leur existence même est en jeu en tant que détentrices séculaires de la seule formule humaniste qui véritablement colle à son objet, l'humanisme chrétien ; parce qu'elles assument, du fait même de l'histoire, des responsabilités envers les autres nations. Les conditions techniques de l'unité du monde sont réalisées. D'ici un siècle, moins peut-être, les peuples vivront à la surface du globe comme les familles d'un même quartier si, du jour au lendemain, elles devaient s'abriter sous. un même toit. À cette unité matérielle doit correspondre une unité spirituelle. Sous l'empire de quelle philosophie s'opérera-t-elle ? Il est facile de dégager les véritables données de l'option. Or, en face d'un Occident ébranlé, tourmenté, inquiet, s'ouvre l'Orient mystérieux, décidé à sortir de son espèce d'immobilité millénaire et dans l'énorme complexe duquel se développent des forces et s'exercent déjà des pressions dont l'explosion pourrait avoir pour le monde des conséquences dont il est proprement impossible à un esprit humain d'imaginer d'avance la profondeur et l'étendue. Or, cet Orient qui n'a pas connu encore le christianisme et à qui il manque la lente initiation au travail scientifique et à l'utilisation des principales créations de la science, va se trouver du jour au lendemain en possession de tous les moyens que la technique moderne, industrielle ou autre, met à la disposition des hommes. Quel usage en fera-til ? Et à quelle conception de l'homme demandera-t-il la pensée directrice de son aménagement technique ? Deux exemples récents, nettement différents, mais également révélateurs, celui du Japon et celui de la Chine, donnent à cette question un commencement de réponse. Les nations occidentales, en tant que créatrices et détentrices des puissances techniques, ne peuvent pas ne pas avoir à l'égard du vas te problème que je viens d'indiquer de très lourdes responsabilités.

L'industrialisation concerne le Canada français au même titre que les autres, parce que lui aussi est issu d'une culture humaniste et chré- 
tienne ; mais elle le concerne plus dangereusement parce qu'il est un peuple faible, encore en voie de formation et que les circonstances historiques l'ont placé dans le rayon d'influence immédiate de la plus grande puissance industrielle de tous les temps et l'ont forcé à participer à la vie commune d'un continent avant d'être en état de décider luimême du moment, de la nature et de l'étendue de sa participation. Considéré du simple point de vue de la politique locale et des relations entre groupements ethniques distincts, il peut paraître indifférent, voire même désirable aux Américains et aux Anglo-Canadiens que le Canada français perde sa personnalité propre dans un complexe humain aux dimensions continentales. Un problème de moins ! Mais considéré dans les perspectives que nous venons d'évoquer d'un monde rigoureusement tenu de repenser l'œuvre de ses mains, à moins d'être détruit par elle, il n'en est pas ainsi. Les antécédents du Canada français, ses traditions, son sens de l'homme, les tendances naturelles de son être et de son histoire sont un actif, non seulement pour luimême, mais pour les populations avoisinantes, et doivent être conservés, sinon dans leur réalité concrète, du moins dans leur inspiration car, dans la conjoncture actuelle du monde, la civilisation occidentale, humaniste et chrétienne, n'a pas les moyens de sacrifier même les moindres de ses forces.

Le régime social du Canada français, tel qu'édifié par les anciennes générations et tel qu'il subsiste encore dans certains centres de notre province, porte deux des caractéristiques de la civilisation particulière dont la nation elle-même procède.

D'abord il est personnaliste, c'est-à-dire ordonné à l'homme, à l'accomplissement de sa vocation. Il embrasse, en les situant chacune à son rang, l'ensemble des valeurs qui y concourent : économiques, intellectuelles, morales, religieuses. Et pour que ce personnalisme ne soit pas un vain mot, mais pour chacun une prérogative vécue, il lui donne comme fondement un régime de travail et un régime de propriété conçus de façon à assurer l'autonomie de l'homme sur le seul plan où initiative et responsabilités personnelles ont une signification et une valeur réelles : celui de la vie privée, de l'activité quotidienne. Les libertés politiques dont on fait de nos jours si grand état n'ont elles-mêmes de sens que si elles procèdent de cette liberté initiale. D'une part, propriété terrienne, source de travail et de subsistance: 
c'est l'établissement agricole ; d'autre part, métier et atelier : c'est l'établissement artisanal. Dans un cas comme dans l'autre, l'homme, maître de ses instruments de travail, a l'initiative de sa vie et en porte la responsabilité. En fait, on peut dire que cet ordre social est d'abord responsable, fondé sur la responsabilité personnelle de l'individu envers lui-même et envers ceux qui dépendent de lui ; et qu'il est libre parce que responsable.

En second lieu, il est communautaire, en ce sens qu'il conçoit l'insertion, selon un mode organique, de l'homme dans la société. Au premier palier, la famille et la profession. En réalisant l'association de la famille, du travail et de la propriété, l'établissement autonome, agricole ou artisanal, fournit l'unité de base de la société. Au second palier, pour remplir les fonctions dont la famille ne peut s'acquitter seule, la paroisse. La paroisse répond à des fins spécifiques d'ordre religieux, mais elle répond aussi à des fins sociales relevant en tout ou en partie du magistère de l'Église : assistance, enseignement. Elle a en outre, au cours de l'histoire, assumé, par carence de l'ancien régime seigneurial, des fonctions économico-sociales qui, bien que n'étant pas de la compétence propre de l'Église, ressortissent néanmoins à sa juridiction dans la mesure où elles touchent à la morale. Ainsi, dès le début de notre histoire, la paroisse s'est instituée en quelque sorte cellule initiale, communauté première de la vie nationale et, par elle, s'affirme au regard du monde le caractère foncièrement spiritualiste de notre civilisation d'origine.

Or, l'évolution politique et l'évolution sociale du Canada français ont suivi des trajectoires différents, voire même contradictoires. La première va dans le sens de l'autonomie pour aboutir au régime fédératif sous lequel nous vivons depuis 1867. Cet affranchissement a pu paraître une conquête définitive. Il n'en est rien. Car l'évolution sociale se produit, elle, dans le sens de l'intégration et tend à assimiler le Canada français au reste du pays et même du continent. De là le conflit entre une pensée politique toujours attachée à l'idée d'autonomie, et une pensée sociale disposée à accepter au moins certaines formes d'intégration.

Depuis la conquête britannique, notre histoire sociale peut être divisée à peu près en trois périodes : l'une qui va de 1763 à1820 envi- 
ron, et durant laquelle le régime économico-social de type paysan et artisanal édifié au temps de la Nouvelle-France continue de suffire à la fois à la subsistance de la population et à l'établissement de son accroissement naturel. Propriétaires de leurs instruments de travail, soustraits à toute dépendance commerciale extérieure, en état de diriger eux-mêmes leur vie communautaire, familiale, professionnelle et paroissiale, les Canadiens français, bien que soumis à une politique d'inspiration étrangère, sont cependant maître chez eux et peuvent continuer leur vie comme si rien d'important ne fût survenu dans leur histoire. Leur milieu réalise la grande condition de son efficacité nationale : il est homogène.

La deuxième période s'étend de 1820 jusque vers 1880. Durant ces soixante années, le vieux régime êconomico-social suffit toujours à la subsistance de la population établie, mais faute d'une politique appropriée de colonisation et de mise en valeur rationnelle des ressources du sol, il ne pourvoit plus à l'établissement complet de l'accroissement naturel. C'est la période de l'émigration vers les États-Unis d'abord, puis vers les autres provinces. Le milieu national conserve son homogénéité, les Canadiens français restent maîtres chez eux, mais ils sont entravés dans leur expansion.

Enfin, la période d'urbanisation et d'industrialisation, l'une portant l'autre. Cette période commence en 1880 et peut être divisée en deux phases : celle du capitalisme libéral, close avec le début du siècle actuel, disons avec la guerre de 1914, phase pendant laquelle l'urbanisation s'effectue à une allure d'apparence normale et pendant laquelle les Canadiens français ont l'air de s'adapter sans trop de difficulté à la vie des villes. Puis, la phase du capitalisme de groupe qui dure toujours et pendant laquelle, par suite de la profonde et rapide transformation de l'économie de la province, se posent le problème économique et le problème social, tels que nous pouvons les observer de nos jours.

Dans les campagnes, lorsque, sous la poussée des pressions extérieures, l'économie paysanne a dû se transformer en économie commerciale, le coopératisme et l'association professionnelle, l'un et l'autre conformes par leur inspiration générale à nos traditions sociales, sont venus organiser la fonction sociale du travail, et ce passage de la simple solidarité familiale des périodes antérieures à la solidarité so- 
ciale des temps actuels s'est effectué dans les cadres et sous l'égide de la paroisse. Preuve que le vieux régime social conçu pour une économie de pionniers, donc nécessairement rurale, possédait néanmoins assez de sève pour se dépasser lui-même, le moment venu, et s'adapter à des circonstances nouvelles.

Dans les villes, il n'en a pas été ainsi. Une économie d'inspiration libérale, donc conçue comme étant à elle-même sa propre loi et sa propre fin, une économie ordonnée au rendement et donc accordant aux techniques la priorité sur l'homme, s'y établissait. Au temps de l'entreprise individuelle et familiale, le désaccord entre la vieille tradition sociale et la pensée dont procédait l'économie nouvelle

ne s'est pas révélé tout de suite dans toute son étendue, et c'est pourquoi durant sa première phase, l'urbanisation n'a pas paru devoir entraîner pour la société canadienne-française de trop graves conséquences, d'autant moins qu'elle se présentait comme remède au mal de l'émigration dont le Canada français était rongé depuis plus d'un demisiècle, Mais le conflit éclate dans toute sa force avec l'avènement de la grande industrie. Les conséquences sont faciles à évaluer. Une population aux quatre-cinquièmes rurale et aux neuf-dixièmes autonome est devenue aux trois-quarts urbaine et aux sept-dixièmes dépendante. Le problème social se présente donc aujourd'hui dans des perspectives complètement différentes.

Quelle attitude prendre à son égard ? Non pas évidemment celle du refus. L'industrie est la vocation de notre sol, une nécessité de notre époque, un besoin de notre population. Et d'ailleurs elle est là ! La seule attitude logique et féconde est celle qui continue de tendre vers l'avenir en retenant du passé ce qui est véritablement exigé par le progrès humain et par la civilisation au sens propre du mot. Or, ce qui, dans notre histoire, a pleine valeur humanisante et civilisatrice, ce ne sont pas les modalités institutionnelles d'un ordre social et politique conçu pour une époque et un milieu différents. Les modalités, fussentelles juridiques ou sociales, sont comme le vêtement : elles s'usent et doivent périodiquement être renouvelées et, selon les besoins repensées. Ce qui dans notre passé social doit être retenu, c'est l'esprit dont procédaient les institutions, les modes de vie. Le personnalisme et le sens communautaire évoqués il y a un instant, issus d'une conception 
spiritualiste de l'homme, sont des valeurs permanentes et universelles qui ont autant de prix pour les populations qui nous entourent qu'elles en ont pour nous-mêmes. Ce sont ces valeurs-là : sens de l'homme et de sa vocation spirituelle, sens de la liberté et de la responsabilité, sens communautaire, familial et professionnel, s'épanouissant dans un véritable sens de la solidarité et de la fraternité sociales, sens de la continuité, sens de la qualité, - ce sont ces valeurs-là, substance même de notre civilisation d'origine, dont il faut faire reprendre conscience à notre population, afin qu'elle aime en vivre et que, spontanément, du seul fait qu'elle en vit, elle les exprime dans des oeuvres originales et les intègre dans un régime institutionnel économique, social et politique qui, adapté à notre époque, n'en reflétera pas moins intensément les véritables richesses de notre histoire.

C'est ce que j'appelais il y a un instant voir les faits par le dedans : faits de l'histoire, faits du moment présent, qui, nous le savons bien, préparent les faits des années à venir. Telle est notre tâche, à nous qui sommes déjà engagés dans la carrière ; à nous surtout à qui incombe la responsabilité de préparer des jeunes aux carrières d'économistes, de sociologues, d'observateurs et de guides de la vie sociale. Dans la conjoncture actuelle de la société et du monde, c'est une tâche très lourde, mais dont la grandeur est aux dimensions mêmes de ses exigences.

\section{Esdras MINVILLE}

Fin du texte 